\title{
Hepatic Secretion of Phospholipid Vesicles in the Mouse Critically Depends on mdr2 or MDR3 P-Glycoprotein Expression
}

\author{
Visualization by Electron Microscopy
}

\begin{abstract}
Aleta R. Crawford, ${ }^{\star}$ Alexander J. Smith, ${ }^{\ddagger}$ Victoria C. Hatch, ${ }^{\S}$ Ronald P.J. Oude Elferink, Piet Borst, ${ }^{\ddagger}$ and James M. Crawford ${ }^{\star}$ *Department of Pathology, Yale University School of Medicine, and Yale Liver Center, New Haven, Connecticut 06520-8023; ${ }^{*}$ Division of Molecular Biology, The Netherlands Cancer Institute, Amsterdam, The Netherlands; ${ }^{\S}$ Division of Respiratory Biology, Harvard School of Public Health, Boston, Massachusetts 02115; and ${ }^{\|}$Department of Gastroenterology and Hepatology, University of Amsterdam, Academisch Medisch Centrum, Amsterdam, The Netherlands
\end{abstract}

\begin{abstract}
Hepatocellular secretion of bile salts into the biliary space induces phospholipid and cholesterol secretion, but the mechanism for integrated lipid secretion is poorly understood. Knockout mice unable to make the canalicular membrane mdr2 P-glycoprotein exhibit normal rates of bile salt secretion, yet are virtually incapable of secreting biliary phospholipid and cholesterol. As the mdr2 P-glycoprotein is thought to mediate transmembrane movement of phospholipid molecules, this mouse model was used to examine the mechanism for biliary phospholipid secretion. In wild-type $m d r 2$ $(+/+)$ mice, ultrarapid cryofixation of livers in situ revealed abundant unilamellar lipid vesicles within bile canalicular lumina. Although $74 \%$ of vesicles were adherent to the external aspect of the canalicular plasma membrane, bilayer exocytosis was not observed. Vesicle numbers in $m d r 2(+I-)$ and $(-I-)$ mice were 55 and $12 \%$ of wild-type levels, respectively. In a strain of $m d r 2(-I-)$ mice which had been "rescued" by heterozygous genomic insertion of the MDR3 gene, the human homologue of the murine $m d r 2$ gene, vesicle numbers returned to $95 \%$ of wild-type levels. Our findings indicate that biliary phospholipid is secreted as vesicles by a process largely dependent on the action of the murine mdr2 P-glycoprotein or human MDR3 P-glycoprotein. We conclude that mdr2-mediated phospholipid translocation from the internal to external hemileaflet of the canalicular membrane permits exovesiculation of the external hemileaflet, a vesiculation process promoted by the detergent environment of the bile canalicular lumen. (J. Clin. Invest. 1997. 100:2562-2567.) Key words: liver $•$ bile $\cdot$ membrane $\cdot$ bile canaliculus • electron microscopy
\end{abstract}

This study was presented in preliminary abstract form at the annual meeting of the American Association for the Study of Liver Diseases, Chicago, November 1995 (1995. Hepatology. 22:316A), and at the Falk International Meeting on Bile Acids, Freiburg, October 1996.

Address correspondence to James M. Crawford, M.D., Ph.D., Department of Pathology, Yale University School of Medicine, 310 Cedar St., P.O. Box 208023, New Haven, CT 06520-8023. Phone: 203785-2784; FAX: 203-737-1064; E-mail: jamesmac.crawford@yale.edu

Received for publication 23 May 1997 and accepted in revised form 1 September 1997.

J. Clin. Invest.

(C) The American Society for Clinical Investigation, Inc. 0021-9738/97/11/2562/06 \$2.00

Volume 100, Number 10, November 1997, 2562-2567

http://www.jci.org

\section{Introduction}

Secretion of bile salts by the liver is critical for elution of phospholipid molecules from the canalicular membrane and for solubilization of free cholesterol in bile (1). Bile salts are amphiphilic steroidal molecules which attain concentrations in excess of $5 \mathrm{mM}$ in the lumina of bile canaliculi, yet do not dissolve the hepatocyte canalicular plasma membrane. Nevertheless, biliary phospholipid secretion is dependent upon the detergent action of luminal bile salts (2). Together bile salts and phospholipid increase the biliary solubility of cholesterol over a million-fold, thereby permitting entry of hepatocellular cholesterol into bile (1). Thus, a critical step in bile formation is the extraction of biliary phospholipid from the canalicular plasma membrane. Although phospholipids appear to be secreted into the canalicular lumen as unilamellar vesicles (3-5), current evidence does not favor a conventional bilayer exocytosis mechanism (6-8) and there is controversy over whether vesicles even form on the canalicular membrane (9-11).

A unique opportunity to gain insight into bile formation is provided by the $m d r 2$ knockout mouse, which is virtually incapable of secreting phospholipid into bile (12). The mdr2 P-glycoprotein is thought to function as a phospholipid translocase ("flippase"), moving biliary-type phosphatidylcholines from the internal to the external hemileaflet of the canalicular membrane $(9,12-15)$. Phosphatidylcholine molecules would then be susceptible to the detergent action of luminal bile salts. Therefore, the purpose of this study was to use imaging techniques to determine whether vesicle formation was proportional to phospholipid secretion in mice heterozygous or homozygously negative for mdr2 P-glycoprotein, and thereby test the hypothesis that vesicle secretion from the external face of the canalicular membrane is the predominant mechanism for phospholipid extrusion into bile.

\section{Methods}

Animals. In our original publication (12), the 129/Ola strain of mouse was used to disrupt the $m d r 2$ gene. However, as 129/Ola mice breed poorly, the mutation was crossed into FVB strain mice, using repeated brother-sister mating until the defect was available in a $>99 \%$ FVB background (16). FVB mice selected for examination were either wild-type $m d r 2(+/+)$, heterozygous $(+/-)$, or homozygously negative $(-/-)$. Because the human MDR3 P-glycoprotein is thought to have the same function as its mouse counterpart, mdr2 $(14,17)$, we also introduced the $M D R 3$ gene into FVB strain $m d r 2$ $(-/-)$ mice, generating two strains, designated A1 and A63. Mice with the human MDR3 P-glycoprotein were generated by cloning a human MDR3 minigene behind an albumin promoter-enhancer construct, and injecting the construct into murine FVB oocytes (17). Two transgenic lines, A1 and A63, were crossed with $m d r 2(-/-)$ mice to generate heterozygous $m d r 2(-/-) M D R 3(+/-)$ mice. RNase pro- 
tection assays of liver RNA demonstrated that MDR3 expression in A63 mice was $\sim 15$-fold higher than in A1 mice. Using a monoclonal antibody (C219) that recognizes all P-glycoprotein subtypes, we found on Western blots a comparable signal in A63 mice and wild-type animals. With the MDR3 specific polyclonal antiserum AVLCL2 (14), the A1 mice demonstrated hepatic MDR3 P-glycoprotein levels that were $15 \%$ of those in A63 mice. No MDR3 mRNA or protein reactivity could be detected in any tissue other than the liver. Mice 3 mo of age at the time of killing were used $(28 \pm 2 \mathrm{~g}, \pm \mathrm{SD}, n=42)$, with no differences in weight between genotypic groups.

Animal procedures. An open laparotomy under pentobarbital anesthesia (50 mg/kg, intraperitoneally) was performed. For physiological studies ( $n=30$ mice, see Fig. 1$)$, the gallbladder was cannulated and bile was collected continuously for $15 \mathrm{~min}$ (12). Bile flow was measured gravimetrically, assuming a specific gravity of 1.00. Bile salts were determined with $3 \alpha$-hydroxysteroid dehydrogenase, phosphatidylcholine using the choline oxidase method, and cholesterol using cholesterol oxidase, as described (12). Phosphatidylcholine detected by the choline esterase method has been shown previously to account for virtually all phospholipid in bile (12).

For ultrastructural studies $(n=12$ mice $)$, an open laparotomy was performed and liver tissue was subjected to ultrarapid cryofixation in situ using a DDK PS1000 ${ }^{\mathrm{TM}}$ device (Delaware Diamond Knives, Inc., Newark, DE), as previously described (6). This procedure involved impelling a freshly cut liver lobe against a highly polished liquid nitrogen-cooled metal block (6), a technique which arrests within milliseconds both biological processes and the biophysical rearrangement of membranes (18). Two specimens from each animal were obtained.

Ultrastructural analysis. Cryofixed liver specimens were subjected to automated freeze-drying and stabilized by fixation at $10^{-3} \mathrm{mbar}$ and $25^{\circ} \mathrm{C}$ with vaporized paraformaldehyde followed by vaporized $\mathrm{OsO}_{4}$, using the LifeCell ${ }^{\circledR}$ MDD-C Molecular Distillation Dryer (RMC Inc., Tucson, AZ) (19). Specimens were infiltrated with degassed Spurr resin, polymerized, and 50-nm thin sections were obtained (two grids containing five thin sections per specimen). Each grid was scanned at magnifications of 250 and 3,000 to identify the cryofixation contact face and the intact cell layers of optimal tissue preservation. All bile canaliculi, defined as luminal structures delimited by intact plasma membranes and tight junctional zones, were identified at a magnification of 7,000, viewed at a magnification of 30,000, and digitized images were obtained. The dimensions and area of bile canaliculi, microvilli, nascent and free vesicles, and structures of uncertain origin were systematically measured as described previously (6).

A rigorous system of blinded analysis was used to avoid observer bias. Section grids were coded and randomized by one individual (V.C. Hatch). All electron microscopy was then performed by a second individual (J.M. Crawford) without knowledge of grid (and hence, mouse) identity. Digitized images of bile canaliculi were then coded and randomized again (by A.K. Ralston), and measured by a fourth individual (A.R. Crawford) without knowledge of image identity. The codes for images and grids were not broken until morphometric analysis of all images was complete. Finally, information about the physiological analysis of animals (performed by R.P.J. Oude Elferink) was withheld until completion of the morphological studies.

The primary structures of interest were canalicular vesicles, as reported in Results. Microvilli were identified in the bile canaliculi of all animals, and there were no significant differences among groups, with 9-13 microvilli on average per canaliculus, occupying between 14 and $20 \%$ of bile canalicular area. Circular to ellipsoid luminal structures with more electron dense interiors were observed, possibly representing vesicles cut in tangential section or microvilli cut in cross section. In our blinded morphometric analysis these were scored as "uncertain," and were found to exhibit no substantive differences among groups, with 1.2 such structures on average per canaliculus. The lack of differences among uncertain structures indicates that they were almost all microvilli cut in cross section.

Statistics. Physiological measurements of bile flow and bile salt, phospholipid, and cholesterol secretion rates were based on three consecutive 5-min collections of bile from each animal. Ultrastructural data were obtained from digital morphometry to determine the area and width of each bile canaliculus, and the dimensions and areas of structural profiles contained within. For each data set, comparisons between experimental animal groups, using mean values obtained for each animal, were made using one-way ANOVA followed by Newman-Keul's multiple range tests for pair-wise comparisons (20), using StatPak software (Northwest Analytical, Portland, OR).

\section{Results}

Physiological parameters are presented in Fig. 1. Bile flow and bile salt secretion rates exhibited no decrements among the $m d r 2(+/+),(+/-)$ and $(-/-)$ mice and two strains of MDR3 rescue mice; the enhanced bile flow in the $m d r 2(-/-)$ mice has been attributed to enhanced ductular fluid secretion (12). Phospholipid secretion rates declined to 78 and $6 \%$ of wildtype in the $m d r 2(+/-)$ and $(-/-)$ mice, respectively. Rescue of the P-glycoprotein defect in the A1 and A63 strains of $m d r 2$ $(-/-) M D R 3(+/-)$ mice raised phospholipid secretion rates to 24 and $113 \%$ of wild-type, respectively. Cholesterol secretion was maintained in the $m d r 2(+/-)$ mice, dropped to near zero in the $m d r 2(-/-)$ mice, and was restored in both strains of $M D R 3$ rescue mice. These findings confirm that biliary phosphatidylcholine secretion is largely dependent upon mdr2 P-glycoprotein function in the mouse. Only a modest rate of phospholipid secretion is required to maintain adequate cholesterol secretion, in keeping with the concept that cholesterol secretion is only indirectly dependent upon mdr2 P-glycoprotein function (21). Although the $m d r 2(-/-)$ MDR3 (+/-) rescue mouse of strain A1 was only marginally different from the $m d r 2(-/-)$ mice, the findings with the $m d r 2(-/-) M D R 3$ $(+/-)$ rescue mice of strain A63 demonstrate that the human MDR3 P-glycoprotein homologue is capable of completely restoring normal rates of biliary phospholipid and cholesterol secretion.

For the ultrastructural studies, 354 bile canaliculi in 12 animals were analyzed, for an average of $\sim 30$ bile canaliculi per animal. Bile canaliculi were readily identified in regions of well-preserved liver parenchyma (within $\sim 30 \mu \mathrm{m}$ of the cryofixation face), as shown in Fig. 2. Canaliculi from all animals contained microvilli, defined as elongate structures with a delimiting membrane in continuity with the canalicular plasma membrane, and a slightly electron-dense interior in continuity with the hepatocyte cytoplasm. Canalicular vesicles were readily observed in $m d r 2(+/+)$ animals (Fig. $2, A$ and $B$ ), with fewer numbers in $m d r 2(+/-)$ animals. Vesicles were defined as membrane-delimited circular to ellipsoid structures with electron-lucent interiors. Most vesicles (478 out of 643) were adherent to the external side of the canalicular membrane, exhibiting abrupt angular junctions and maintaining a membranous barrier between the vesicle interior and the hepatocyte cytoplasm (Fig. 2, $A$ and $B$, arrowheads). Most canaliculi from the $m d r 2(-/-)$ mice were devoid of vesicles (Fig. 2, $C$ and $D)$. However, a small, but significant, number was still observed in these animals (Fig. $2 E$ ), with features identical to those seen in $m d r 2(+/+)$ and $m d r 2(+/-)$ animals (Fig. $2 F)$. Vesicles were identified in low numbers in the canaliculi of the A1 MDR3 rescue mouse. In contrast, abundant vesicles were present in the bile canaliculi of the A63 MDR3 rescue mice (Fig. 2, G and $H$ ).

The key results of our morphometric analysis are presented 

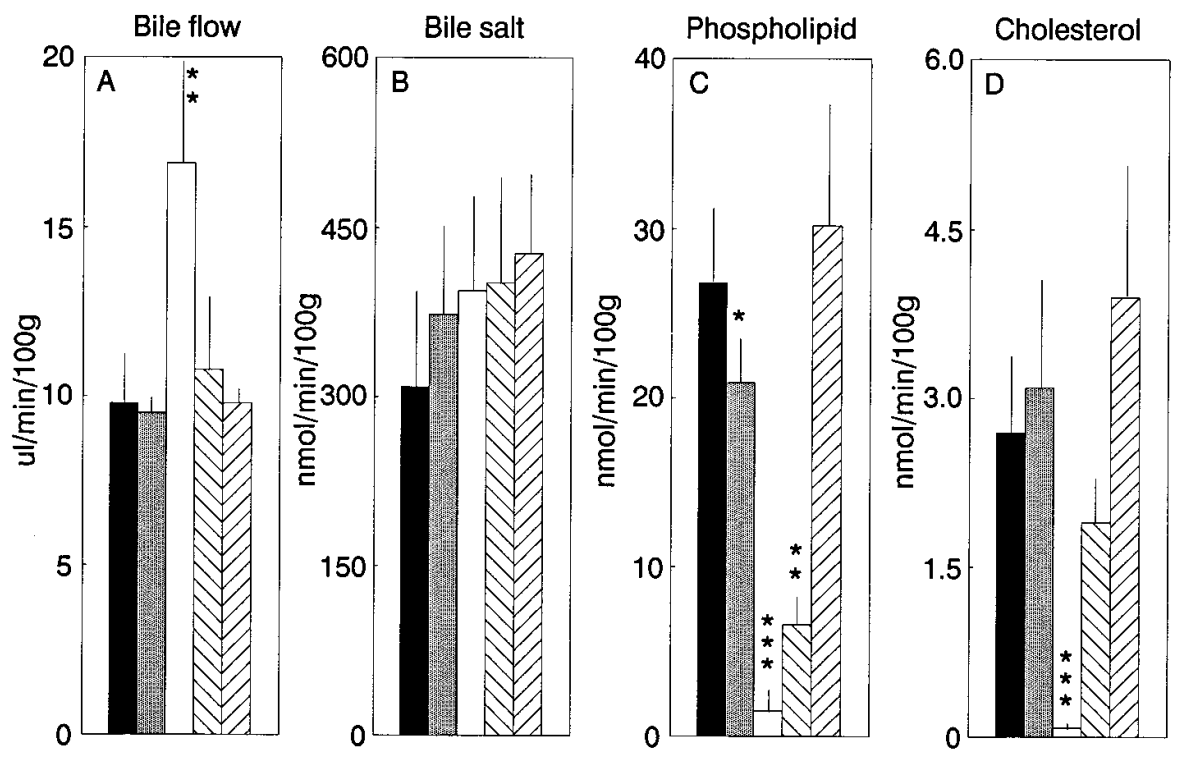

Figure 1. Bile secretion parameters. Bile flow $(A)$ and secretion rates (mean $\pm \mathrm{SD})$ for bile salts $(B)$, phospholipid $(C)$, and cholesterol $(D)$ are shown for mice with a wild-type $m d r 2(+/+)$ genotype $(n=7$, closed bars), heterozygous (+/-) genotype ( $n=7$, gray bars), homozygous genotype $(-/-)(n=7$, open bars $)$, and MDR3 "rescue" genotype [strain A1: $m d r 2(-/-)$ $M D R 3(+/-), n=5$, left diagonal bars; strain A63: $m d r 2(-/-), M D R 3(+/-), n=$ 4 , right diagonal bars]. $* P<0.05$ vs. wildtype; $* * P<0.01$ vs. wild-type; $* * * P<$ 0.001 vs. wild-type.

in Fig. 3 in three ways: the percentage of bile canaliculi containing vesicles $(A)$, the mean number of vesicles per canaliculus $(B)$, and the mean percentage of the cross-sectional area of each canaliculus occupied by vesicles $(C)$. All three panels demonstrate that vesicles were relatively abundant in wildtype $m d r 2(+/+)$ mice, at intermediate values in $m d r 2(+/-)$ mice, and minimal values in $m d r 2(-/-)$ mice. In the A1 strain $M D R 3$ rescue mouse examined, vesicles were only minimally restored, whereas in the A63 strain MDR3 rescue mice there was almost complete normalization of vesicle numbers. There were no significant differences in microvilli among the animals.

Canalicular vesicles were not spheroidal. The mean diameters of all 643 vesicles were $75 \pm 14 \mathrm{~nm}$ (minimum) by $103 \pm 20$ (maximum, $\pm \mathrm{SD}$ ). The mean axial ratio (minimum/maximum) was significantly less than unity $(0.79 \pm 0.19, P<0.0001)$, with no significant differences for vesicles adherent to the canalicular membrane or free within the lumen. Vesicle orientation was unrelated to the direction of cryofixation, so that mechanical distortion is an unlikely source of the ellipsoid morphology.
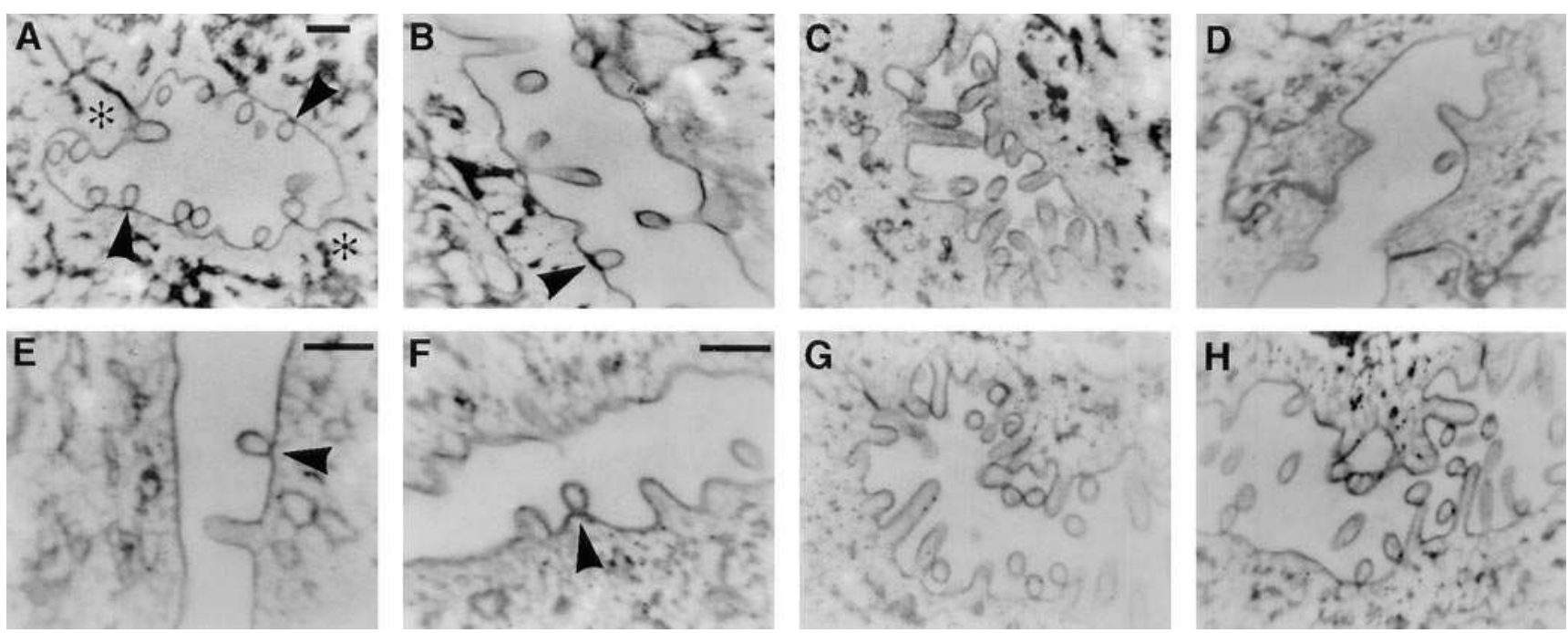

Figure 2. Electron spectroscopic imaging of bile canaliculi from cryofixed mouse liver. $(A)$ Bile canaliculus from an $m d r 2(+/+)$ mouse delineated by tight junctional regions (asterisks), showing the presence of multiple vesicles with zones of adhesion (arrowheads) to the canalicular membrane. (B) Longitudinal section of a bile canaliculus from an $m d r 2(+/+)$ mouse, showing microvilli cut in cross section, and an adherent vesicle (arrowhead). ( $C$ and $D$ ) Bile canaliculi from $m d r 2(-/-)$ mice, containing microvilli but no vesicles. $(E)$ Bile canaliculus from an $m d r 2$ $(-/-)$ mouse, clearly containing a microvillus and an adherent vesicle (arrowhead); the vesicle exhibits the characteristic electron-lucent interior and zone of membrane fusion with the external aspect of the canalicular plasma membrane. $(F)$ Bile canaliculus from an $m d r 2(+/-)$ mouse, showing microvilli and an adherent vesicle (arrowhead). ( $G$ and $H$ ) Bile canaliculi from $m d r 2(-/-)$ MDR3 (+/-) rescue mice of strain A63, containing microvilli and abundant adherent lipid vesicles. Original magnification for $A, B, C, D, G$, and $H$ : $\times 30,000$; bar $=200 \mathrm{~nm}$. Original magnification for $E$ and $F: \times 50,000$; bar $=200 \mathrm{~nm}$. 

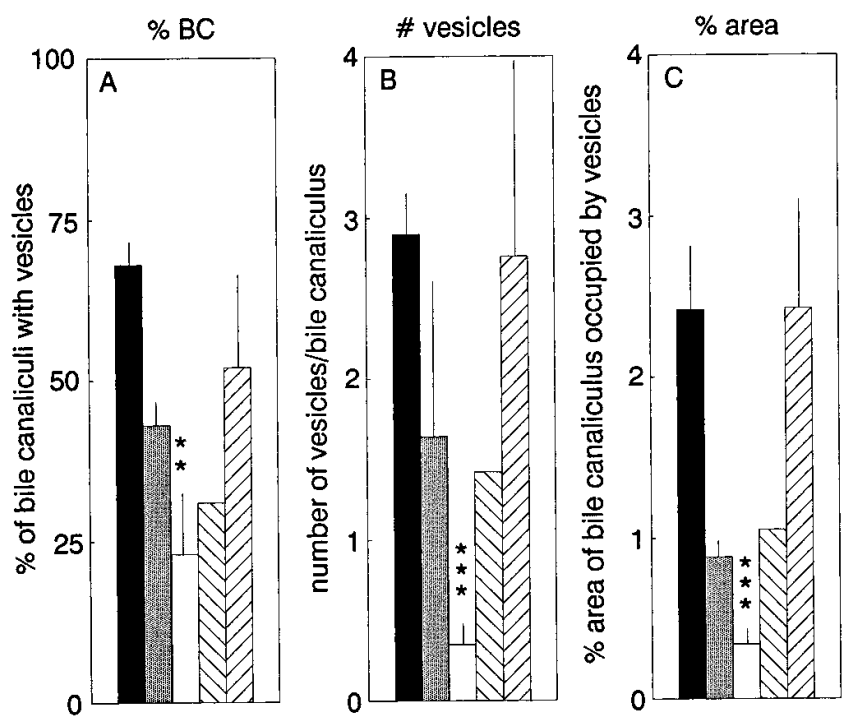

Figure 3. Morphometric analysis of bile canalicular vesicles. The percentage of bile canaliculi containing vesicles $(A)$, average number of vesicles per canaliculus $(B)$, and average percent area of each canaliculus occupied by vesicles $(C)$ are shown for mice with a wild-type $m d r 2(+/+)$ genotype $(n=4, \pm \mathrm{SEM}$, closed bars $)$, heterozygous $(+/-)$ genotype $(n=2, \pm$ difference, gray bars $)$, homozygous genotype $(-/-)(n=4, \pm$ SEM, open bars $)$, and MDR3 "rescue" genotype [strain A1: $m d r 2(-/-)$ MDR3 $(+/-), n=1$, left diagonal bars; strain A63: $m d r 2(-/-), M D R 3(+/-), n=2, \pm$ difference, right diagonal bars]. $* * P<0.01$ vs. wild-type; $* * P<0.001$ vs. wild-type.

Rather, for the 478 adherent vesicles observed, 406 (84\%) were oriented with their long axis perpendicular to the canalicular membrane. Moreover, the zone of membrane fusion between adherent vesicles and the canalicular membrane exhibited a thickness $(27 \pm 6 \mathrm{~nm}$, range $16-60 \mathrm{~nm})$ which was significantly greater $(P<0.01)$ than that of the surrounding canalicular membrane $(14 \pm 3 \mathrm{~nm})$ or the apical dome of the adherent vesicles $(15 \pm 6 \mathrm{~nm})$.

\section{Discussion}

Our findings confirm that the action of the mdr2 P-glycoprotein or its human homologue, MDR3, is essential for secretion of phosphatidylcholine into bile. The key conclusion from this study is that once phosphatidylcholine molecules are translocated from the internal to the external hemileaflet by the mdr2 (or MDR3) P-glycoprotein, they are eluted as unilamellar vesicles into bile. Interestingly, restructuring of a membrane hemileaflet into a newly formed bilayer vesicle also has been proposed as the mechanism for shedding of the phosphatidylcholine surface monolayer of circulating lipoproteins (22, $23)$. Conversely, coating of the alveolar surface by lung surfactant requires restructuring of secreted multilamellar arrays of membrane bilayers to form hemileaflet monolayers at the airtissue interface (24). In the case of the liver, hepatocellular secretion of bile salts into the bile canalicular lumen is necessary for elution of phospholipid vesicles from the canalicular membrane (2), presumably on the basis of their detergent interactions with the external hemileaflet (7).

Our observations permit the consideration of a working model for biliary lipid secretion (Fig. 4). First, the action of the mdr2 P-glycoprotein may generate transient fluid microdomains of biliary-type phosphatidylcholines in the external hemileaflet of the membrane, an otherwise sphingomyelin/ cholesterol rich membrane which is resistant to the detergent actions of bile salts (25). The relative position of the mdr2 P-glycoprotein to putative microdomains (in the center or around the perimeter) is currently unknown. Second, bile salts secreted into the lumen through the action of the canalicular bile acid transporter (cBAT) attain millimolar concentrations in the bile canalicular lumen. These bile salts will interact preferentially with the cholesterol-poor fluid membrane microdomains composed of biliary-type phosphatidylcholines, relative to the surrounding cholesterol-sphingomyelin-rich canalicular membrane (26). Because the efficiency of bile salt secretion is maintained in animals that lack the mdr2 P-glycoprotein, it is probable that cBAT resides in the bulk canalicular membrane, as depicted. We cannot exclude the possibility that this transporter lies in close approximation to mdr2 P-glycoproteins, when present. Third, asymmetric accumulation of bile salts and phosphatidylcholine molecules in the external hemileaflet will generate a local increase in lateral pressure and focal bulging, thereby promoting exovesiculation. Such focal events may be accentuated by the shear resistance of the surrounding sphingomyelin-rich canalicular membrane (27), particularly since sphingomyelin is capable of interdigitating across the membrane bilayer (28). Fourth, the distinct angular junction between the sides of adherent vesicles and the canalicular membrane (Fig. 2, $E$ and $F$ ) and the thickness of the adhesion zone raise the possibility that lipid phase transitions occur within the external hemileaflet of the membrane. Phase transitions through hexagonal lipid phases are predicted by the ternary phase diagram for phosphatidylcholine hydration in the presence of bile salts (29), as would occur during vesiculation of the canalicular plasma membrane (7). Further experimental work will be required to determine whether such phase transitions occur in situ in the canalicular membrane. Finally, the nonspherical shape of vesicles, with the consistently decreased curvature of the lateral aspects of adherent vesicles, may have relevance to cholesterol entry into bile. Lateral diffusion of cholesterol into nascent cholesterol-poor phospholipid vesicles may "stiffen" their sides. We postulate that this is a potential mechanism for entry of cholesterol into bile, since biliary phospholipid vesicles are thought to be important carriers of cholesterol in bile (30). Indeed, the minimal amount of phospholipid secretion present in the A1 rescue mice is capable of restoring cholesterol secretion (Fig. 1). However, luminal bile salts are capable of solubilizing cholesterol from the canalicular membrane even in the absence of phospholipid secretion $(21,31)$. Thus, multiple mechanisms for cholesterol secretion may be operative, in particular lateral diffusion from the canalicular membrane into nascent vesicles and/or bile salt-mediated transfer of cholesterol through the aqueous phase of bile to luminal vesicles $(32,33)$.

Although the singular role of mdr 2 in biliary phospholipid secretion has been questioned $(34,35)$, the source of the very low phospholipid secretion rates and vesicle secretion we observed in $m d r 2(-/-)$ mice (Figs. 1 and 3 ) is not established. It should be noted that an alternative strain of $m d r 2(-/-)$ mice did not exhibit any residual phospholipid secretion (12). Thus, further studies will be required to determine whether additional mechanisms may be present for phosphatidylcholine translocation across the canalicular membrane bilayer (36). 


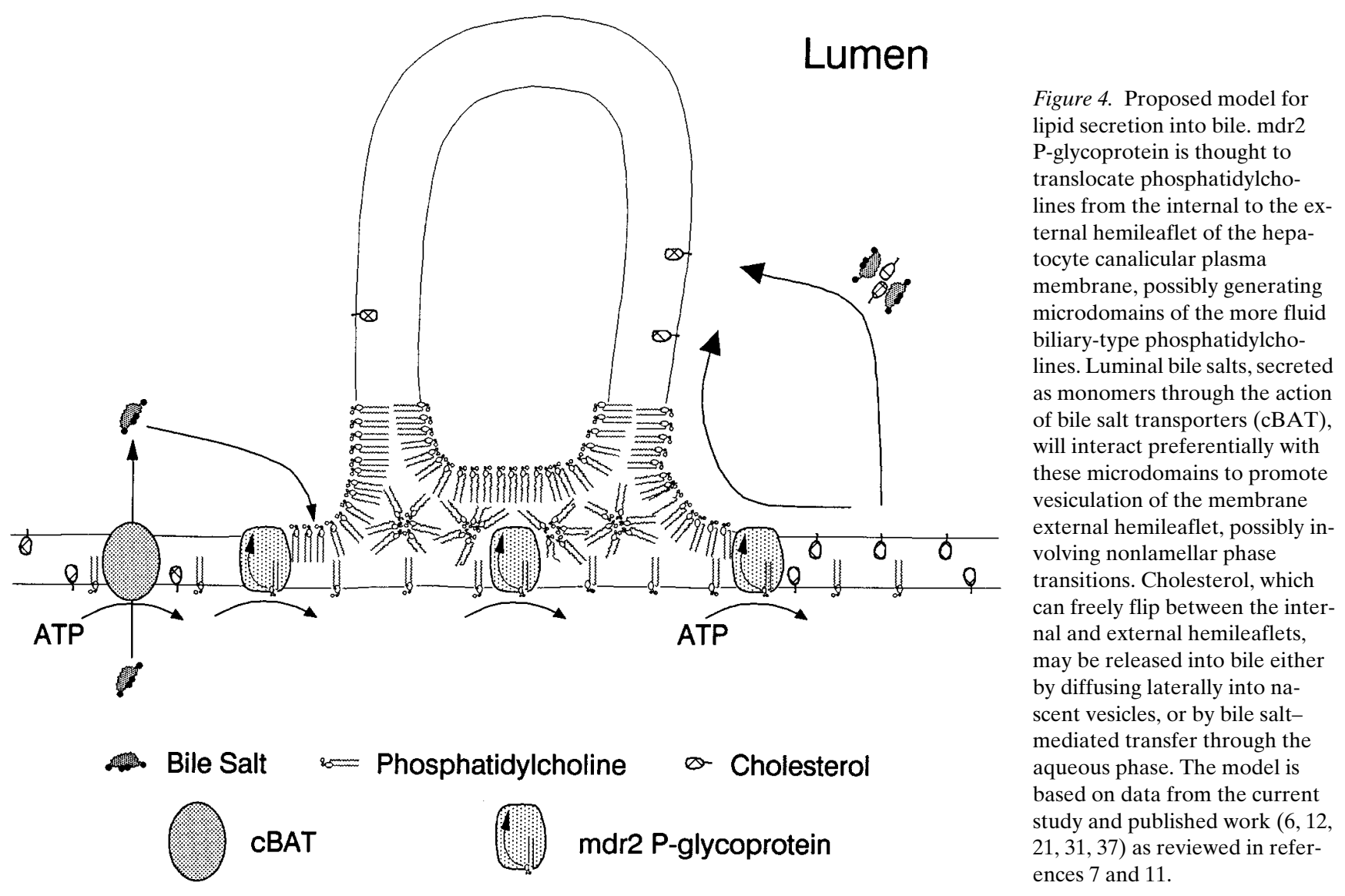

In conclusion, we have demonstrated that phospholipid is secreted as vesicles into bile, and that vesicle secretion is almost wholly dependent upon mdr2 or MDR3 P-glycoprotein function. Our findings provide strong support for the concept that biliary phosphatidylcholine molecules are flipped from the internal to the external hemileaflet of the hepatocyte canalicular membrane by the action of murine mdr2 P-glycoprotein or human MDR3 P-glycoprotein, followed by release of phosphatidylcholine vesicles from the external hemileaflet into bile. Vesiculation of the external hemileaflet provides an explanation of how luminal bile salts can extract large quantities of phospholipid on the basis of their detergent action, without disrupting the integrity of the detergent-resistant canalicular plasma membrane. This remarkable mechanism for selective secretion of membrane phospholipids appears to be uniquely adapted to the detergent environment of the bile canaliculus.

\section{Acknowledgments}

Thanks are given to Amy K. Ralston for expert technical assistance, to Dr. Martin C. Carey for critical discussions during the course of this study, and to Dr. John J. Godleski for expert advice and use of the Electron Microscopy Facility, Division of Respiratory Biology, Harvard School of Public Health, Boston, MA. The experiments reported herein were conducted according to the principles set forth in the Guide for the Care and Use of Laboratory Animals, Institute of Animal Resources, National Research Council.

This study was supported by National Institutes of Health grants DK-44981 and DK-39512 (to J.M. Crawford), National Institutes of Health grant DK-34854 (to the Membrane Core Facility, Harvard Di-

gestive Diseases Center), grants NKI 88-6 and NKI92-41 from the Dutch Cancer Society (to P. Borst), and by a program grant from the Netherlands Foundation for Scientific Research (NWO 902-23-097, to R.P.J. Oude Elferink and P. Borst).

\section{References}

1. Carey, M.C., and J.T. LaMont. 1992. Cholesterol gallstone formation. I. Physical-chemistry of bile and biliary lipid secretion. Prog. Liv. Dis. 10:139-163.

2. Verkade, H.J., R.J. Vonk, and F. Kuipers. 1995. New insights into the mechanism of bile acid-induced biliary lipid secretion. Hepatology. 21:11741189.

3. Cohen, D.E., L.S. Leighton, and M.C. Carey. 1992. Bile salt hydrophobicity controls vesicle secretion rates and transformations in native bile. Am. J. Physiol. 263:G386-G395.

4. Gilat, T., and G.J. Sömjen. 1996. Phospholipid vesicles and other cholesterol carriers in bile. Biochim. Biophys. Acta. 1286:95-115.

5. Möckel, G., S. Gorti, R.K. Tandon, R. Tanaka, and M.C. Carey. 1995. Microscope laser light scattering spectroscopy of vesicles within canaliculi of rat hepatocyte couplets. Am. J. Physiol. 269:G73-G84.

6. Crawford, J.M., G.-M. Möckel, A.R. Crawford, S.J. Hagen, V.C. Hatch, S. Barnes, J.J. Godleski, and M.C. Carey. 1995. Imaging biliary lipid secretion in the rat: ultrastructural evidence for vesiculation of the hepatocyte canalicular membrane. J. Lipid Res. 36:2147-2163.

7. Crawford, J.M. 1996. The role of vesicle-mediated transport pathways in hepatocellular bile secretion. Semin. Liver Dis. 16:169-190.

8. Groen, A.K., R.P.J. Oude Elferink, and J.M. Tager. 1996. Control analysis of biliary lipid secretion. J. Theor. Biol. 182:427-436.

9. Ruetz, S., and P. Gros. 1995. Enhancement of mdr2-mediated phosphatidylcholine translocation by the bile salt taurocholate: implications for hepatic bile formation. J. Biol. Chem. 270:25388-25395.

10. Nies, A.T., Z. Gatmaitan, and I.M. Arias. 1996. ATP-dependent phosphatidylcholine translocation in rat liver canalicular plasma membrane vesicles. J. Lipid Res. 37:1125-1136.

11. Oude Elferink, R.P.J., G.N.J. Tytgat, and A.K. Groen. 1997. The role of mdr2 P-glycoprotein in hepatobiliary lipid transport. FASEB (Fed. Am. Soc. 
Exp. Biol.) J. 11:19-28

12. Smit, J.J.M., A.H. Schinkel, R.P.J. Oude Elferink, A.K. Groen, E. Wagenaar, L. Van Deemter, C.A.A.M. Mol, R. Ottenhoff, N.M.T. Van der Lugt, M.A. Van Roon, et al. 1993. Homozygous disruption of the murine $m d r 2$ P-glycoprotein gene leads to a complete absence of phospholipid from bile and to liver disease. Cell. 75:451-462.

13. Ruetz, S., and P. Gros. 1994. Phosphatidylcholine translocase: a physiological role for the mdr2 gene. Cell. 77:1071-1081.

14. Smit, J.J.M., A.H. Schinkel, C.A.A.M. Mol, D. Majoor, W.J. Mooi, A.P.M. Jongsma, C.R. Lincke, and P. Borst. 1994. Tissue distribution of the human MDR3 P-glycoprotein. Lab. Invest. 71:638-649.

15. Van Helvoort, A., A.J. Smith, H. Sprong, I. Fritzche, A.H. Schinkel, P. Borst, and G. Van Meer. 1996. MDR1 P-glycoprotein is a lipid translocase of broad specificity, while MDR3 P-glycoprotein specifically translocates phosphatidylcholine. Cell. 87:507-517.

16. Van Nieuwkerk, C.M.J., R.P.J.O. Elferink, A.K. Groen, R. Ottenhoff, G.N.J. Tytgat, K.P. Dingemans, M.A.V.B. Weerman, and G.J.A. Offerhaus. 1996. Effects of ursodeoxycholate and cholate feeding on liver disease in FVB mice with a disrupted $m d r 2$ P-glycoprotein gene. Gastroenterology. 111:165-171.

17. Smith, A.J., J.L.P.M. Timmermans-Hereijgers, B. Roelofsen, K.W.A. Wirtz, W.J. van Blitterswijk, J.J.M. Smit, A.H. Schinkel, and P. Borst. 1994. The human MDR3 P-glycoprotein promotes translocation of phosphatidylcholine through the plasma membrane of fibroblasts from transgenic mice. FEBS Lett. 354:263-266.

18. Baatsen, P.H.W.W. 1993. Empirically determined freezing time for quick-freezing with a liquid-nitrogen-cooled copper block. J. Microsc. 172:71-79.

19. Crawford, J.M., S. Barnes, R.C. Stearns, C.L. Hastings, and J.J. Godleski. 1994. Ultrastructural localization of a fluorinated bile salt in hepatocytes. Lab. Invest. 71:42-51.

20. Zar, J. 1974. Biostatistical Analysis. Prentice-Hall, Inc., Englewood Cliffs, NJ.

21. Oude Elferink, R.P.J., R. Ottenhoff, M. van Wijland, J.J.M. Smit, and A.H. Schinkel. 1995. Regulation of biliary lipid secretion by mdr2 P-glycoprotein in the mouse. J. Clin. Invest. 95:31-38.

22. Tall, A.R., and D.M. Small. 1980. Body cholesterol removal: role of plasma high-density lipoproteins. Adv. Lipid Res. 17:1-51.

23. Handa, T., H. Saito, and K. Miyajima. 1990. Phospholipid monolayers at the triolein-saline interface: production of microemulsion particles and conversion of monolayers to bilayers. Biochemistry. 29:2884-2890.

24. Hall, S.B., Z. Wang, and R.H. Notter. 1994. Separation of subfractions of the hydrophobic components of calf lung surfactant. J. Lipid Res. 35:13861394.

25. Crawford, J.M. 1996. Intracellular trafficking and plasma membrane se- cretion of small organic solutes involved in hepatocellular bile formation. Comp. Biochem. Physiol. 115:341-354.

26. Cohen, D.E., M. Angelico, and M.C. Carey. 1990. Structural alterations in lecithin-cholesterol vesicles following interactions with monomeric and micellar bile salts: physical-chemical basis for subselection of biliary lecithin species and aggregative states of biliary lipids during bile formation. J. Lipid Res. 31:55-70.

27. Almeida, P.F.F., W.L.C. Vaz, and T.E. Thompson. 1992. Lateral diffusion and percolation in two-phase, two-component lipid bilayers. Topology of the solid-phase domains in-plane and across the lipid bilayer. Biochemistry. 31: 7198-7210.

28. Levin, I.W., T.E. Thompson, Y. Barenholz, and Y. Huang. 1985. Two types of hydrocarbon chain interdigitations in sphingomyelin bilayers. Biochemistry. 24:6282-6286.

29. Small, D.M., M.C. Bourgès, and D.G. Dervichian. 1966. The biophysics of lipidic association. I. The ternary systems lecithin-bile salt-water. Biochim. Biophys. Acta. 125:563-580.

30. Yuet, P.K., D. Blankschtein, and J.M. Donovan. 1996. Ultracentrifugation systematically overestimates vesicular cholesterol levels in bile. Hepatology. 23:896-903.

31. Oude Elferink, R.P.J., R. Ottenhoff, M. van Wijland, C.M.G. Frijters, C. van Nieuwkerk, and A.K. Groen. 1996. Uncoupling of biliary phospholipid and cholesterol secretion in mice with reduced expression of mdr2 P-glycoprotein. J. Lipid Res. 37:1065-1075.

32. Vlahcevic, Z.R., E.C. Gurley, D.M. Heuman, and P.B. Hylemon. 1990. Bile salts in submicellar concentrations promote bidirectional cholesterol transfer (exchange) as a function of their hydrophobicity. J. Lipid Res. 31:1063-1071.

33. Robins, S.J., and J.M. Fasulo. 1997. High density lipoproteins, but not other lipoproteins, provide a vehicle for sterol transport to bile. J. Clin. Invest. 99:380-384.

34. Berr, F., P.J. Meier, and B. Stieger. 1993. Evidence for the presence of a phosphatidylcholine translocator in isolated rat liver canalicular plasma membrane vesicles. J. Biol. Chem. 268:3976-3979.

35. Kullak-Ublick, G.A., T. Gerloff, B. Hagenbuch, F. Berr, P.J. Meier, and B. Stieger. 1996. Expression of a rat liver phosphatidylcholine translocator in Xenopus laevis oocytes. Hepatology. 23:1254-1259.

36. Cohen, D.E., and M. Fuchs. 1996. Transmembrane translocation of phosphatidylcholines and biliary lipid secretion: evidence for "flippases" in the canalicular plasma membrane. Hepatology. 24:1546-1548.

37. Oude Elferink, R.P.J., R. Ottenhoff, M. van Wijland, C.M.G. Frijters, C van Nieuwkerk, and A.K. Groen. 1996. Uncoupling of biliary phospholipid and cholesterol secretion in mice with reduced expression of mdr2 P-glycoprotein. J. Lipid Res. 37:1065-1075. 